\title{
EFECTO DE LA TEMPERATURA EN LAS ETAPAS DE REFINADO Y MEZCLADO SOBRE EL CONTENIDO DE POLIFENOLES DE UN CHOCOLATE BLANCO
}

\section{TEMPERATURE EFFECT ON THE POLYPHENOLS OF REFINED AND MIXED PROCESS OF A WHITE CHOCOLATE}

\author{
Ing. Carlos Duque Acevedo*, MsC. Luz Caballero Pérez ${ }^{* *}$ \\ PhD. Maria Esther Rivera ${ }^{* *}$ \\ * Servicio Nacional de Aprendizaje SENA, Grupo de Investigación y Desarrollo del \\ Cacao y Chocolatería. \\ Kilómetro2 vía Palogordo vereda Guatiguara, Piedecuesta, Santander, Colombia. \\ 0576800600 . \\ E-mail: * caidu@misena.edu.co. \\ - Xzzz *Universidad de Pamplona, Facultad de Ingenierías y Arquitecturas. \\ Maestría en Ciencia y Tecnología de Alimentos. Grupo de Investigaciones, \\ GIBA. \\ Km 1 Vía Bucaramanga, Campus Universitario, Pamplona, Norte de Santander, \\ Colombia. \\ 0575685303 \\ E-mail: ** luzcaballero@unipamplona.edu.co,** maes@unipamplona.edu.co
}

Resumen: El objetivo de la presente investigación fue el de evaluar el efecto de la temperatura y tiempo de exposición en las etapas de refinado y mezclado sobre el contenido de polifenoles en un chocolate blanco. Se utilizaron extractos de polifenoles de la variedad FVS 41, definiendo 4 tratamientos para las formulaciones de chocolate blanco: (T1: $0 \%$; T2: $0.1 \%$, T3: $0.3 \%$ y T4: $0.8 \%$ ) trabajadas a temperaturas de 40 a 50 $\pm 1{ }^{\circ} \mathrm{C}$ y tiempos de exposición de 5,10 y $15 \mathrm{~min}$. Al chocolate blanco se le evaluó el contenido de polifenoles totales, empleando el método Folin - Ciocalteu (F-C). Los resultados mostraron que no existen diferencias estadísticamente significativas entre la temperatura y tiempo de exposición a un nivel de significancia de 0.05 . Se concluye que el efecto de la temperatura y tiempo de exposición en las etapas de refinado y mezclado en la elaboración de chocolate blanco fue leve, siendo más notoria la disminución del contenido de polifenoles $(0,10 \mathrm{mg}$ AG / g muestra) en el tratamiento 4, a mayor temperatura y tiempo de exposición $\left(50 \pm 1^{\circ} \mathrm{C}\right.$ y $\left.15 \mathrm{~min}\right)$.

Palabras clave: Chocolate blanco, Cacao, Efecto, Polifenoles, Temperatura.

Abstract: The research objective was to evaluate the effect of temperature and exposure time in the refining and mixing stages on the content of polyphenols in a white chocolate. The extracts of polyphenols of the FVS 41 variety was were, defining 4 treatments for white chocolate formulations (T1: $0 \%$; T2: $0.1 \%$, T3: $0.3 \%$ y T4: $0.8 \%$ ) worked at temperature of 40 to $50 \pm 1{ }^{\circ} \mathrm{C}$ and exposure times 5,10 and $15 \mathrm{~min}$. To the white 
chocolate was evaluated the polyphenols totals content, through the Folin-Ciocalteu method (F-C). The results showed that there are no statically significant differences between the temperature and exposure time at a significance level of 0,05. It is conclude that the effect of the temperature and exposure time in the stages of refined and mixed in the elaboration of white chocolate was slight, the decrease of the polyphenols content $(0,10 \mathrm{mg} \mathrm{AG} / \mathrm{g}$ sample) in the treatment 4 , to higher temperature and exposure time (50 $\pm 1{ }^{\circ} \mathrm{C}$ and $\left.15 \mathrm{~min}\right)$.

Keywords: White chocolate, Cocoa, Effect, Polyphenols, Temperature.

\section{INTRODUCCIÓN}

Los polifenoles son un conjunto heterogéneo de moléculas que comparten la característica de poseer en su estructura varios grupos bencénicos sustituidos por funciones hidroxílicas (Hernández y Prieto, 1999). Aunque la mayor capacidad antioxidante de la dieta está en frutas y vegetales y se la proporcionan el contenido en vitaminas E, C y $\beta$-carotenos, (Ayola et al., 2019), también los polifenoles contribuyen de manera importante, (Delgado et al., 2018) pues sus anillos aromáticos con sustituyentes hidroxilos les brindan una estructura especialmente adecuada para ejercer una acción antioxidante al poder actuar como donadores de hidrógenos o electrones o servir como atrapadores de radicales libres (Nakatani et al., 2000).

Entre los polifenoles, los flavonoides constituyen el grupo más importante e incluye a más de 5.000 compuestos bien identificados (Harborne et al., 2000). Todos poseen una estructura de 3 anillos consistentes en 2 centros aromáticos (anillos A y B) y un heterociclo oxigenado central (anillo C) y están típicamente conjugados a azúcares 9, clasificándose en 6 subgrupos: flavonoides, flavonas, flavanonas, isoflavonas, antocianinas y catequinas (Gee y Johnson, 2001).

Los compuestos fenólicos constituyen uno de los grupos de sustancias más numerosas y ampliamente distribuidas en el reino vegetal, con más de 8000 estructuras ampliamente conocidas y pueden ser divididos en al menos 10 clases diferentes dependiendo de su estructura básica, estas clases son: fenoles simples, benzoquinonas, ácidos fenólicos, acetofenonas, ácidos fenialaceticos, ácidos hidroxiaminicos, fenilpropanos, cumarinas e isocumarinas, antraq uinonas y los flavonoides (Wollgast et al., 2000).

La polifenol oxidasa (PPO), Es una enzima involucrada en la oxidación de los polifenoles presentes en diferentes plantas y frutos. Cuando las células de los tejidos vegetales se encuentran sanas e intactas, la PPO y los sustratos (fenoles) se encuentran separados y ubicados en los cloroplastos y vacuolas respectivamente; pero cuando ocurre cualquier alteración, los fenoles y la PPO se juntan e inician las reacciones de oxidación (Badia et al., 2004).

En los granos de cacao, la activación de la PPO es ocasionada por el incremento en la temperatura y por el cambio en las condiciones de $\mathrm{pH}$ que ocurren durante los procesos de fermentación y secado. Como consecuencia de la activación de la enzima, en presencia de oxígeno, ocurre la oxidación de los flavonoides, disminuyendo así la astringencia y el sabor amargo del cacao (Bonilla et al., 2019; Jinap et al., 2004).

Pese a que la calidad sensorial del cacao aumenta después de los procesos de oxidación que ocurren en el beneficio, el contenido de polifenoles disminuye, afectando significativamente el valor funcional del cacao. Con el fin de obtener semillas de cacao (Theobroma cacao L.) con un elevado contenido de polifenoles y evitar los procesos de oxidación, se propuso la inactivación de la PPO en las semillas frescas, mediante un tratamiento térmico de escaldado. El grano obtenido mediante este tratamiento, aunque es amargo y astringente, se puede utilizar como materia prima en la industria farmacéutica y en la producción de suplementos dietarios y alimentos funcionales (Vera et al., 2017; Duque et al., 2015; Wollgast y Anklam, 2000).

Los flavonoides son los polifenoles más abundantes en el cacao. El chocolate es rico en flavonoides con la estructura de las catequinas y epicatequinas y sobre todo de los polímeros tipo procianicidas que se forman durante el procesamiento del grano de cacao (Theobroma cacao L.) por unión desde 2 a 10 monómeros de 
epicatequina debido a la acción en esas condiciones de la enzima polifenol oxidasa (Baba et al., 2007).

Las procianidinas formadas por la unión de 2 a 6 monómeros de epicatequina son las más abundantes, mientras que las que contienen entre 2 y 5 monómeros son las formas más activas, probablemente debido a que la forma monomérica es metabolizada muy rápidamente y excretada, mientras las poliméricas mayores de 6 unidades pueden tener dificultades para penetrar las membranas celulares y son por tanto pobremente absorbidas (Weisburger et al., 2001).

Las catequinas y procianidinas aisladas del cacao (Theobroma cacao L.) tienen fuertes propiedades antioxidantes in vitro, como lo demuestra al comparar las catequinas del chocolate con las del té, con respecto a las cuales muestran un efecto antioxidante 4 veces mayor, (Arts et al., 1999). Siendo las catequinas y sus oligómeros unidos por enlace $\mathrm{C} 4 \rightarrow \mathrm{C} 8$ las de mayor efecto (Osakabe et al., 2002).

Los Flavonoles, se caracterizan por poseer un grupo ceto en el carbono $\mathrm{C}_{4}$ y una instauración entre los carbonos $\mathrm{C}_{2}$ y $\mathrm{C}_{3}$. Poseen además un grupo hidroxilo adicional en el carbono $\mathrm{C}_{3}$. La quercetina es el compuesto más representativo, estos compuestos se localizan principalmente en el tejido externo y aéreo de la planta. La distribución y la concentración de los flavonoles pueden ser distinta incluso en frutas procedentes de la misma planta, Pueden aparecer como monómeros o como polímeros con distintos grados de polimerización.

Los flavanoles más representativos en los alimentos son de tipo flavan-3-ol, y estos pueden aparecer como monómeros (catequinas), como dímeros condensados entre sí y como oligómeros (procianidinas), o bien pueden aparecer como polímeros (proantocianidinas o taninos condensados) (Quiñones et al., 2012).

Epicatequina y catequina son los compuestos mayoritarios en frutas. Las catequinas también se encuentran en el vino y en el chocolate, que son las fuentes mayoritarias. En cambio, galocatequina, epigalocatequina y epigalocatequina galato aparecen principalmente en el té. Los polifenoles que se conservan en los cotiledones, son transformados mediante oxidación hacia quinonas, mediado por las Polifenol-Oxidasas (PPO), que como antes se dijo reduce el contenido de polifenoles (astringencia) y permite el desarrollo de color marrón (Vázquez et al., 2016).
A partir de los antecedentes mencionados se planteó el objetivo general de la presente investigación: evaluar el efecto de la temperatura y tiempos de exposición en las etapas de refinado y mezclado sobre el contenido de polifenoles totales en la elaboración de un chocolate blanco, con el fin de ofrecer una alternativa de consumo.

\section{METODOLOGIA}

\section{MATERIALES}

Para la elaboración de un chocolate blanco con adición de polifenoles, se utilizaron las siguientes materias primas; manteca de cacao desodorizada, de casa Luker, azúcar blanca, leche en polvo entera y descremada de Colanta, lecitina de soya para disminuir la viscosidad, vainillina para dar sabor de empresa Silesia y polifenoles de cacao FSV41.

Material vegetal FSV 41. clon de Cacao (Theobroma cacao L.) proveniente del municipio El Playón, desarrollados en la Sede Aguas Calientes del SENA.

El proceso de obtención de polifenoles se realizó en el Centro de Investigación en Ciencia y Tecnología de Alimentos laboratorio CICTA de la Universidad Industrial de Santander UIS, con el método de extracción con dilapidación, determinación del perfil de ácidos grasos, extracción sin deslipidación y liofilización.

Polifenoles añadidos en la formulación (chocolate blanco). Se procedió a añadir los polifenoles extraídos (Theobroma cacao L.) de la variedad Fedacacao San Vicente (FSV 41). La dosis empleada de polifenoles se determinó según parámetros de la normatividad: Resolución 1511 de 2011 norma para el chocolate y los productos de chocolate CODEX STAN 87-1981, Rev. 1-2003 alimentario y Resolución 4124 de 1991, tomando como referencia la cantidad de polifenoles contenida en un chocolate con leche y chocolate oscuro, se revisó parámetros máxima utilizada con antioxidantes artificiales (BHT, BHA, BTHQ) cuya formulación está entre 100 - $200 \mathrm{mg} / \mathrm{kg}$.

Se realizaron 3 repeticiones del proceso de elaboración de chocolate blanco, hasta estandarizar las cantidades por balance de materia de polifenoles utilizando a las siguientes concentraciones T1: $0 \%, \mathrm{~T} 2: 0.1 \%$, T3: $0.3 \%$ y T4: $0.8 \%$ de polifenoles, para que el producto final estuviera acorde con los requerimientos específicos de antioxidantes bajo la Resolución 4124 de 1991. 
En esta etapa se midieron los polifenoles por el método Folin-Ciocalteu, Determinando cual fue la muestra con polifenoles con mejor estabilidad y cantidad de antioxidantes.

A continuación, se describen los métodos empleados en la determinación de polifenoles:

\section{a. Ensayo de Folin-Ciocalteu (FC).}

El contenido de Polifenoles Totales (PT) de los extractos liofilizados y sin liofilizar se determinó siguiendo el procedimiento descrito por Wollgast (2004). El ensayo se realizó por triplicado a cada muestra. Brevemente, $50 \mu \mathrm{l}$ del extracto a valorar, $1,5 \mathrm{ml}$ de reactivo de Folin-Ciocalteu (diluido 10 veces en agua destilada) y $1,5 \mathrm{ml}$ de solución de $\mathrm{Na} 2 \mathrm{CO} 3(7.5 \%$ p/v) se mezcló vigorosamente. $\mathrm{La}$ mezcla se dejó en reposo durante 60 min protegida de la luz. Luego, se midió la absorbancia de las muestras a $765 \mathrm{~nm}$ en un espectrofotómetro (GENESYS 20, Thermo Spectronic). El contenido de PT se expresó en mg equivalentes de Ácido Gálico (mgEAG) por gramo de muestra seca $(\mathrm{g})$.

\section{b. Determinación de la actividad antioxidante mediante el método ORAC (Capacidad de absorción de radicales de oxigeno)}

El mecanismo de reacción se basa en la transferencia de un átomo de hidrogeno del antioxidante al radical libre, para ello se emplea un indicador que genera el radical piróxilo (ROO). En presencia de un compuesto antioxidante, el radical forma un enlace con un átomo de hidrogeno del compuesto antioxidante y se originan un hidroperóxido $(\mathrm{ROOH})$ y un radical antioxidante estable. Este ensayo es en ejemplo del rompimiento de reacciones en cadena por transferencia de un átomo de hidrogeno proveniente de un compuesto antioxidante y sirve para medir la degradación oxidativa de una molécula fluorescente (fluoresceína) (Ou et al., 2001). (Niño, AB 2018).

La fluoresceína reacciona con el AAPH (2,2-azobis (2-amidino-propano) dihidrocloruro) el cual es un azo derivado generador de radicales libre. El AAPH produce radicales piróxilo por calentamiento, que dañan la molécula fluorescente, la cual se oxida para neutralizar los radicales, resultando en pérdida de su fluorescencia. Los antioxidantes protegen la molécula fluorescente de la degeneración oxidativa y el grado de protección se cuantifica usando un espectrofluorimetro.
La velocidad en la disminución de la fluorescencia puede reducirse en presencia de un antioxidante, ya que este compite con el compuesto fluorescente para estabilizar el radical. $\mathrm{La}$ capacidad antioxidante se determina por la disminución en la fluorescencia y la cantidad de producto formado en función del tiempo. La protección del antioxidante se mide a partir del área bajo la curva de la muestra y del blanco de reacción. La diferencia de estos valores se extrapola en la curva de calibración de Trolox. Esta metodología puede ser adaptada para la detección de antioxidantes tanto hidrófilos como hidrofóbicos (Ou et al., 2001).

\section{c. Contenido de polifenoles totales.}

Se determinó mediante el ensayo de FolinCiocalteu (F-C), siguiendo el procedimiento descrito por Wollgast. El contenido de polifenoles se expresa como equivalentes de ácido gálico (mg ÁG/g de muestra). El análisis cuantitativo se realizó por el método del estándar externo y para ello, se construyó una curva de calibración con ácido gálico $(10.0-100,0 \mu \mathrm{g} / \mathrm{mL})$ con el fin de expresar los resultados en términos de Equivalentes de Ácido Gálico (GAE).

\section{ANÁLISIS ESTADÍSTICO DE DATOS.}

Los datos obtenidos fueron analizados mediante el software estadístico SPSS v. 22, aplicando el análisis de varianza a un nivel de significancia de 0.05 , con el fin de determinar el efecto de las variables independientes (temperatura y tiempo de exposición sobre el contenido de polifenoles en un chocolate blanco.

\section{RESULTADOS}

\subsection{Efecto de la temperatura y el tiempo de exposición sobre el contenido de polifenoles añadidos.}

En la figura 1, se observa una disminución del $69,23 \%$ (0,25 mg ácido gálico / g muestra) del contenido de polifenoles totales inicial $0,65 \mathrm{mg}$ ácido gálico / g muestra contenido en el chocolate blanco ( $\mathrm{T}$ 1), esta disminución se presenta en tiempos cortos de exposición de 5, 10 y 15 minutos a temperaturas entre 42 y $49{ }^{\circ} \mathrm{C}$, quedando con una concentración final de $0,45 \mathrm{mg}$ ácido gálico/g muestra. 


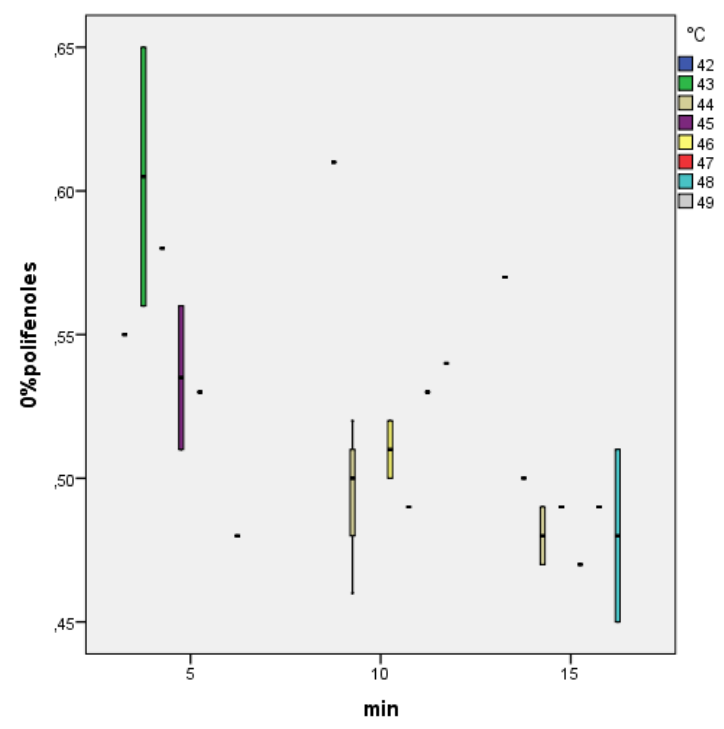

Figura 1. Efecto de la temperatura y el tiempo de exposición sobre el contenido de polifenoles en el tratamiento 1

En la figura 2 se observa una disminución del 0,10 mg ácido gálico / g muestra del contenido de polifenoles totales inicial $0,85 \mathrm{mg}$ ácido gálico / g muestra contenido en el chocolate blanco con el $0.1 \%$ de polifenoles extraídos ( $\mathrm{T} 2$ ). Esta disminución se presenta en mayor proporción a medida que se incrementan los tiempos de exposición de 5, 10 y 15 minutos a temperaturas entre 42 y $49^{\circ} \mathrm{C}$, quedando con una concentración final de $0,75 \mathrm{mg}$ ácido gálico/g muestra.

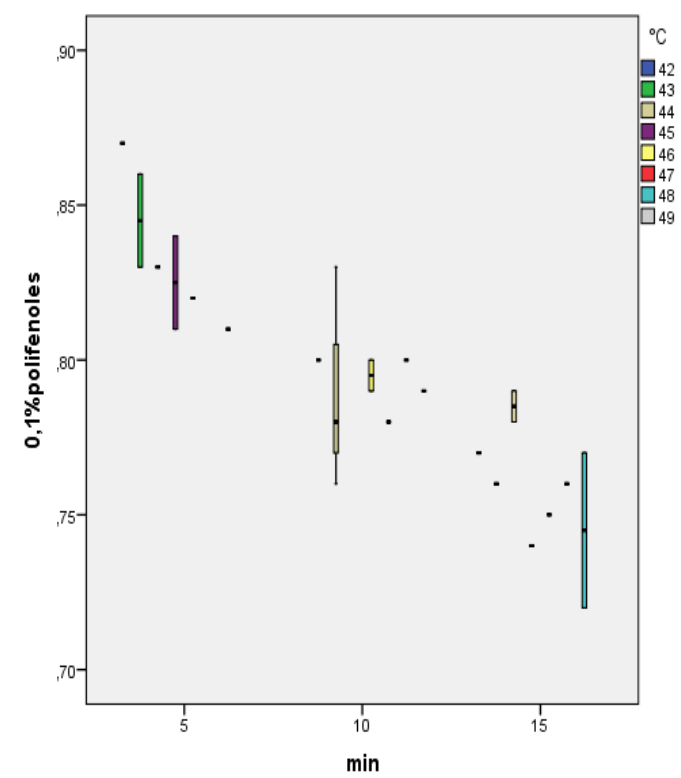

Figura 2. Efecto de la temperatura y el tiempo de exposición sobre el contenido de polifenoles en el tratamiento 2 .
De la figura 2 se infiere que existen cambios en la concentración de polifenoles en el tratamiento 2, por el efecto de la temperatura y el tiempo de exposición, coincidiendo con los resultados obtenidos en el estudio realizado por Di Mattia et al., (2014) quien reportó que la actividad antioxidante y la concentración de polifenoles del chocolate se reducen significativamente por el efecto de la temperatura en los procesos de conchado. Además, teniendo en cuenta que en estudios realizados por Ioannone et al., (2015) quienes observaron que el tiempo y temperatura afectan la reducción de polifenoles, en tiempos cortos.

El chocolate blanco con el $0,3 \%$ de extractos de polifenoles ( $\mathrm{T} 3$ ), alcanzo valores entre 0,948 a 0,865 mg ácido gálico / g muestra del contenido de polifenoles totales para los tiempos de exposición de 5, 10 y 15 minutos, en los rangos de temperatura de 42 a $49{ }^{\circ} \mathrm{C}$ respectivamente, observando una disminución de la concentración inicial $0,10 \mathrm{mg}$ ácido gálico/g de muestra (figura 3).

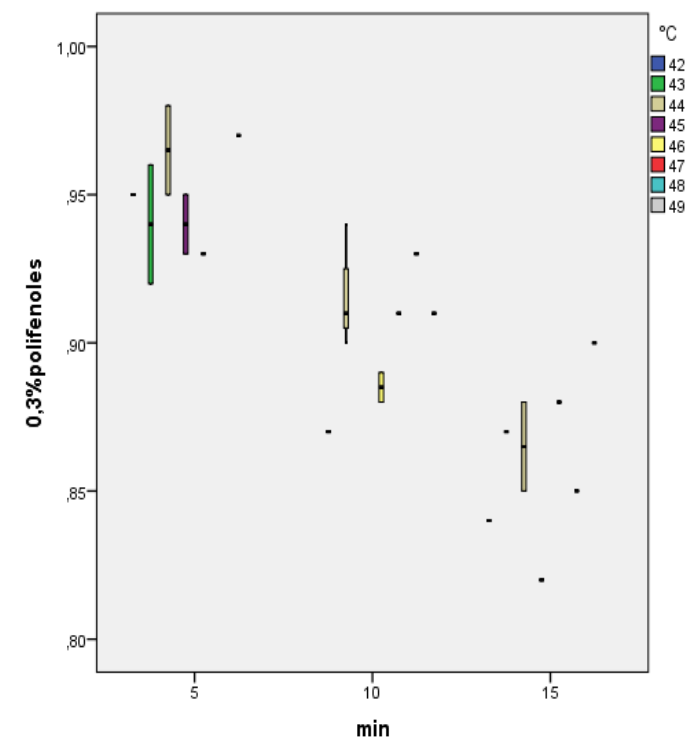

Figura 3. Efecto de la temperatura y el tiempo de exposición sobre el contenido de polifenoles en el tratamiento 3

De la figura 3 se infiere que existe un cambio en la concentración de polifenoles inicial en los primeros 5 minutos y a medida que aumenta el tiempo de exposición (15 minutos) y la temperatura, quedando con $0,90 \mathrm{mg}$ ácido gálico / g muestra del contenido de polifenoles totales en el chocolate blanco con extractos con una concentración de 0,8 $\%$. 


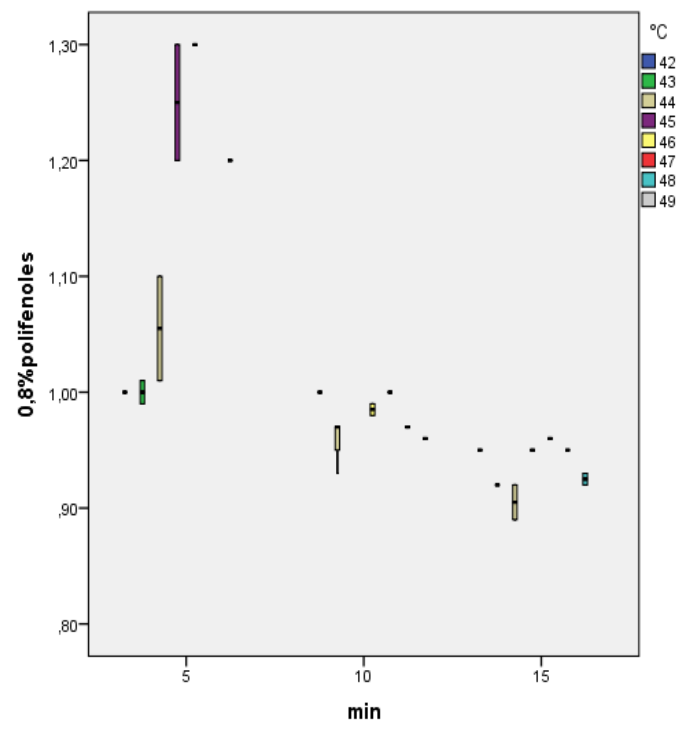

Figura 4. Efecto de la temperatura y el tiempo de exposición sobre el contenido de polifenoles en el tratamiento 4.

En la figura 4, se observa un cambio en la concentración de polifenoles inicial en los primeros 5 minutos y a medida que aumenta el tiempo de exposición (15 minutos) y la temperatura, quedando con $0,90 \mathrm{mg}$ ácido gálico / g muestra del contenido de polifenoles totales en el chocolate blanco con extractos con una concentración de 0,8 $\%$.

En la Tabla 1 se observa que la variación en el contenido de polifenoles no fue estadísticamente significativo $(0,982 \geq 0,05)$.

Tabla 1. Análisis de varianza de la temperatura en los tratamientos aplicados

\begin{tabular}{|c|c|c|c|c|c|}
\hline Fuente & \begin{tabular}{|l|} 
Suma de \\
cuadrados
\end{tabular} & $\begin{array}{c}\text { Grados de } \\
\text { libertad }\end{array}$ & $\begin{array}{c}\text { Media } \\
\text { cuadrática }\end{array}$ & Fc & $p$-valor \\
\hline \begin{tabular}{|l} 
Entre \\
grupos
\end{tabular} & 0,001 & 2 & 0,001 & 0,018 & 0,982 \\
\hline \begin{tabular}{|l|}
$\begin{array}{l}\text { Dentro de } \\
\text { grupos }\end{array}$ \\
\end{tabular} & 4,030 & 105 & 0,038 & & \\
\hline Total & 4,031 & 107 & & & \\
\hline
\end{tabular}

De la figura 5 se infiere que, a mayor tiempo de exposición, se presenta una disminución de la concentración de polifenoles. Se observa que a una temperatura de $50{ }^{\circ} \mathrm{C}$ y un tiempo de exposición de 15 minutos tiene un efecto sobre el contenido de polifenoles añadidos en el chocolate blanco con una concentración $0,8 \%$ (T4) disminuyendo 0.35 mg ácido gálico/ g muestra, mientras que los tratamientos 1,2 y 3 presentaron cambios mínimos a los 5,10 y 15 minutos.

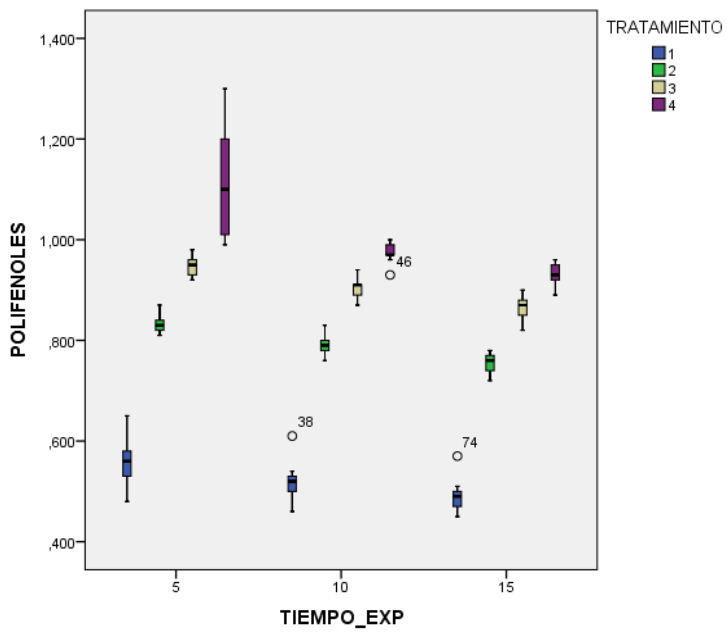

Figura 5. Efecto del tiempo de exposición sobre el contenido de polifenoles en los tratamientos

Los anteriores resultados son coherentes con los resultados obtenidos en las investigaciones de Acevedo et al., (2017), quienes evidenciaron que la temperatura del proceso de conchado es una variable que incide en el contenido de polifenoles y directamente en la capacidad antioxidante de la cobertura de chocolate semi amargo generando una disminución a condiciones térmicas elevadas. Otros estudios evidencian que no hay cambios sobre el contenido total de polifenoles en la etapa posterior al refinado donde la adición es la etapa de estabilización de los antioxidantes Cadena y Herrera (2008). Los principales efectos sobre la reducción de polifenoles y capacidad antioxidante dados durante el conchado se presentaron a temperatura de $60{ }^{\circ} \mathrm{C}$, en el proceso de refinado y mezclado para chocolate blanco las temperaturas varían desde 40 a $50{ }^{\circ} \mathrm{C}$, donde no se apreciaron cambios significativos en los rangos de toma de muestras cada 5 minutos.

Estudios sobre la cuantificación de polifenoles realizados por Abdul et al., (2014) reportan que la actividad antioxidante podría disminuir debido al cambios de temperatura., Wollgast (2004) y Caligiani et al., (2007), han demostrado que el contenido de catequina ha sido menor al contenido de Epicatequina, lo cual coincide con los resultados obtenidos en la presente investigaciones, donde se determinó que a $50{ }^{\circ} \mathrm{C}$ de temperatura y 15 minutos de exposición disminuye el contenido de polifenoles. 


\section{CONCLUSIONES}

- $\quad$ Se determinó que a mayor temperatura y mayor tiempo de exposición, el chocolate blanco presenta una leve disminución en el contenido de polifenoles $(0,10 \mathrm{mg}$ ácido gálico / g muestra) estadísticamente no significativa.

Se concluyó que las temperaturas evaluadas $\left(40\right.$ a $\left.50{ }^{\circ} \mathrm{C}\right)$ y tiempos de exposición de 5,10 y 15 minutos, en las etapas de refinado y mezclado no presentan un efecto estadísticamente significativo sobre el contenido de polifenoles adicionados en el chocolate blanco a concentraciones del $0,1 \%, 0,3 \%$ y $0,8 \%$.

\section{RECONOCIMIENTO}

Los autores agradecen al grupo de investigación GIBA, de la Universidad de Pamplona y al Servicio Nacional de Aprendizaje SENA, Grupo de Investigación y Desarrollo del Cacao y Chocolatería por su apoyo y aportes para el desarrollo de la investigación.

\section{REFERENCIAS}

Abdul, K.; Azlan, A.; Ismail, A.; Hashim, P.; Abdullah, N. (2014). Antioxidant properties of cocoa pods and shells. Malaysian Cocoa Journal 8: 49-56.

Acevedo, L., Mejía, D., Acosta, E., Valencia, W. y Penagos, L. (2017). Efecto de la temperatura del conchado sobre los polifenoles en un chocolate semi-amargo. Revista Alimentos Hoy, 25(41), 31-50. https://acta.org.co/acta_sites/alimentoshoy/ind ex.php/hoy/article/view/447

Arts, I., Hollman, P., y Kromhout, D. (1999). Chocolate as a source of tea flavonoids. The Lancet, 354 (9177), 488. DOI:https://doi.org/10.1016/S01406736(99)02267-9

Ayola C. Yoicelin, Maldonado M. Lida, Yanza H. Erick, Maldonado O. Yohanna. (2019). Efecto de la liofilización en betacarotenos del guacamole. Revista@limentech, Ciencia y Tecnología Alimentaria. ISSN 1692-7125. Volumen $17 \mathrm{~N}^{\circ}$ 2. Pp: 6 - 25 .

Baba, S., Osakabe, N., Kato, Y., Natsume, M., Yasuda, A., Kido, T. y Kondo, K. (2007). Continuous intake of polyphenolic compounds containing cocoa powder reduces LDL oxidative susceptibility and has beneficial effects on plasma HDL-cholesterol concentrations in humans. The American journal of clinical nutrition, 85(3), pp. 709717. https://doi.org/10.1093/ajcn/85.3.709

Bonilla R., María J. y Vera R., José M. (2019) Características fisicoquímicas y colorimétricas de licores de cacao obtenidos de los clones TCS 06, FEAR 5 Y FSV 41. Revista @ limentech, Ciencia y Tecnología Alimentaria. ISSN 1692-7125. Volumen 17 $\mathrm{N}^{\circ}$ 1. Pp: 40 -59.

Cadena C. Tatiana y Herrera A. Mayerly. (2008). Evaluación del efecto del procesamiento del cacao sobre el contenido de polifenoles y su actividad antioxidante. Trabajo de grado para optar el titulo de Química. Universidad Industrial de Santander. Pp 91.

Caligiani, A., Cirlini, M., Palla, M., Ravaglia, R., Arlonio, M., GC-MS (2007). Detectión of ciral Markers in cocoa Beans of Different Quality and Geographic Origin. En Chilarity. Vol 19; p.329-334. https://doi.org/10.1002/chir.20380

Delgado, J. D., Mandujano, J. I., Reátegui, D., y Ordoñez, E. S. (2018). Desarrollo de chocolate oscuro con nibs de cacao fermentado y no fermentado: polifenoles totales, antocianinas, capacidad antioxidante y evaluación sensorial. Scientia Agropecuaria, $9(4), \quad 543-550 . \quad$ DOI https://doiorg.bdigital.sena.edu.co/10.17268/sci.agropec u.2018.04.10

Di Mattia, C., Martuscelli, M., Sacchetti, G., Beheydt, B. y Mastrocola, D. (2014). Effect of Different Conching Processes on Procyanidin Content and Antioxidant Properties of Chocolate. Food Research International 63, 367-

https://doi.org/10.1016/j.foodres.2014.04.009

Duque A., Carlos I., Arrieta S., Alexander., y Torres, Alexandra. (2015). Influencia del contenido de manteca de cacao en las propiedades térmicas de muestras de chocolates por medio TGA y DSC modificando las concentraciones de grasa. Revista@limentech, Ciencia y Tecnología Alimentaria. ISSN 1692-7125. Volumen 13, $\begin{array}{lllll}\mathrm{N}^{\circ} & 2, & \mathrm{pp}: & 154 & -162 .\end{array}$ DOI: https://doi.org/10.24054/16927125.v2.n 2.2015.1884

Gee, J. y Johnson, I. (2001). Polyphenolic compounds: interactions with the gut and implications for human health. Current medicinal chemistry, 8(11), 1245-1255. DOI: https://doi.org/10.2174/0929867013372 256. 
Harborne, J. B., y Williams, C. A. (2000). Advances in flavonoid research since 1992. Phytochemistry, 55(6), 481-504. https://doi.org/10.1016/S00319422(00)00235-1

Hernández Á, Maureen, y Prieto González, Elio A. (1999). Plantas que contienen polifenoles: Antioxidantes dentro del estilo de vida. Revista Cubana de Investigaciones Biomédicas, 18(1), 12. Recuperado en 14 de julio de 2020, de http://scielo.sld.cu/scielo.php?script=sci_artte xt\&pid=S086403001999000100004\&lng=es\&tlng=es.

Ioannone, F., Di Mattia, C., De Gregorio, M., Sergi, M., Serafini, M. y Sacchetti, G. (2015). Flavanols, Proanthocyanidins and Antioxidant Activity Changes during Cocoa (Theobroma Cacao L.) Roasting as Affected by Temperature and Time of Processing. Food Chemistry, $\quad 174, \quad 256-\quad 62$. https://doi.org/10.1016/j.foodchem.2014.11.01 9

Jinap, S., Jamilah, B., y Nazamid, S. (2004). Effect of polyphenol concentration on pyrazine formation during cocoa liquor roasting. Food Chemistry, 85(1), 73-80. https://doi.org/10.1016/j.foodchem.2003.06.00 5

Nakatani, N. (2000). Phenolic antioxidants from herbs and spices. Biofactors, 13(1-4), 141146.

Niño, AB (2018). Microturbina Pelton, una solución real de energía para zonas no interconectadas (ZNI). Revista Tecnologías de Avanzada, ISSN: 1692-7257.

Osakabe, N., Yasuda, A., Natsume, M., Sanbongi, C., Kato, Y., Osawa, T., y Yoshikawa, T. (2002). Rosmarinic acid, a major polyphenolic component of Perilla frutescens, reduces lipopolysaccharide (LPS)-induced liver injury in D-galactosamine (D-GalN)-sensitized mice. Free Radical Biology and Medicine, 33(6), 798-806. https://doi.org/10.1016/S08915849(02)00970-X

Ou, B., Hampsch, M. y Prior, R. (2001). Development and validation of an improved oxygen radical absorbance capacity assay using fluorescein as the fluorescent probe. J Agric Food Chem 49, 4619 - 4626. https://doi.org/10.1021/jf010586o.

Quiñones, M., Miguel, M., y Aleixandre, A. (2012). Los polifenoles, compuestos de origen natural con efectos saludables sobre el sistema cardiovascular. Nutrición Hospitalaria, 27(1), 76-89. DOI:10.3305/nh.2012.27.1.5418.

Singleton, V. \& Rossi, J. A. (1965). Colorimetry of Total Phenolics With PhosphomolybdicPhosphotungstic Acid Reagents. Am. J. Enol. Vitic. 16, 144-158.

Singleton, V., Orthofer, R. \& Lamuela-Raventós, R. (1999). Analysis of Total Phenols and Other Oxidation substrates and Antioxidants by Means of Folin-Ciocalteu Reagent. Methods in Enzymology 299, 152-179

Vázquez, A., Ovando, I., Adriano, L., Betancur, D., y Salvador, M. (2016). Alcaloides y polifenoles del cacao, mecanismos que regulan su biosíntesis y sus implicaciones en el sabor y aroma. Archivos Latinoamericanos de Nutrición, 66(3), 239-255. Recuperado de: http://search.ebscohost.com.bdigital.sena.edu. co/login.aspx ?direct=trueydb=lthyAN $=11797$ 1808ylang=esysite=ehost-live

Vera R. José M.; Arrieta S. Alexandre; Quintana Lucas F.; García J. Alberto. (2017). Evaluación de las propiedades fisicoquímicas como parámetros de calidad en la fermentación de clones de Cacao CCN51, TSC01. Revista @limentech, Ciencia y Tecnología Alimentaria. ISSN 1692-7125. Volumen $\quad 15 \quad \mathrm{~N}^{\circ} \quad$ 2. $\mathrm{Pp}$ : $76 \quad-86$. DOI: https://doi.org/10.24054/16927125.v2.n 2.2017.2970

Weisburger, J. (2001). Chemopreventive effects of cocoa polyphenols on chronic diseases. Experimental Biology and Medicine, 226(10), 891-897. https://doi.org/10.1177/153537020122601003

Wollgast, J. (2004). The contents and effects of polyphenols in chocolate, Qualitative and quantitative analices of polyphenols in chocolate and chocolate raw products as well as evaluation of potencial implications of chocolate in human health. Alemania. (Trabajo de doctorado). Universidad de Gieben, Alemania.

Wollgast, J. y Anklam, E. (2000). Review on polyphenols in Theobroma cacao: changes in composition during the manufactured of chocolate methodology for identification and quantification. Food Res Int, 33, 423-447. https://doi.org/10.1016/S09639969(00)00068-5 